\title{
On numerically predicting the onset and mode of instability in atomistic systems
}

\author{
Phanisri P. Pratapa and Phanish Suryanarayana ${ }^{* a}$ \\ ${ }^{a}$ College of Engineering, Georgia Institute of Technology, GA 30332, USA
}

\begin{abstract}
We present an accurate and efficient method based on the Lanczos algorithm for predicting the onset and mode of instability in atomistic systems. Specifically, we develop a framework that is identically applicable to all flavors of atomistic simulations, including ab-initio calculations. Notably, we do not make any apriori assumptions regarding the nature of the instability or its location. We verify the accuracy of the proposed approach by studying defect nucleation during the nanoindentation of a triangular lattice and hydrostatic tension test of an Aluminum crystal. We demonstrate that the computational cost in practical calculations scales linearly with system size, and is accompanied by a small prefactor. Overall, the proposed method is attractive because it enables the stability analysis of atomistic systems at the mesoscale.
\end{abstract}

Key words: Instability, Lanczos method, Linear scaling, Defect nucleation, Nanoindentation, Hydrostatic tension test.

\section{Introduction}

The chemistry of the core, long ranged elastic fields, and discrete nature of the lattice enable crystal defects to have a significant impact on the macroscopic properties of solids $[1,2]$. This motivates the accurate characterization of defects, which includes developing a fundamental understanding of the mechanisms involved in their nucleation. Since ab-initio calculations are limited by their large computational cost $[3,4,5,6,7,8]$, atomistic simulations based on empirical potentials have been the preferred choice for studying the nucleation of defects $[9,10,11,12,13$, $14,15,16,17]$. However, these studies focus on the observation rather than prediction of defect nucleation. In particular, the nature of the instabilities - characterized by a sudden rearrangement of atoms resulting in either a structural transformation, phase transition or nucleation of a defect - which give rise to crystal defects is relatively less well understood. Though the present work is targeted towards the atomistic scale, de-

\footnotetext{
${ }^{*}$ Corresponding Author

ish.suryanarayana@ce.gatech.edu)

Preprint submitted to Elsevier
}

fect nucleation related phenomena can also be studied at higher length scales [18].

In view of the above discussion, there have been previous efforts to predict the onset and mode of instability in atomistic systems [19, 20, 21, 22]. However, these approaches employ a complete eigendecomposition of the force constants matrix, i.e., Hessian matrix. Since the computational and memory costs of such methods scale cubically and quadratically with matrix size, respectively, the size of systems that can be studied is severely limited. To overcome this bottleneck in the case of defect nucleation, it has been proposed that the stability analysis be restricted to a suitably chosen subset of atoms [23, 24, 25]. However, this strategy assumes the instability mode to be localized, and requires apriori knowledge of the center and size of the localization region to be efficient. Another previously developed technique associates defect nucleation with the loss of positive definiteness of the atomic scale acoustic tensor [26, 27, 28]. This requires redefining continuum level quantities at the atomic scale, which can be challenging $[29,30]$. Furthermore, the instability mode cannot be calculated, and the analysis needs to be

April 22, 2016 
performed for every atom, which can make it inefficient.

In this work, we develop a framework based on the Lanczos method for accurately and efficiently predicting the onset and mode of instability in atomistic systems. Notably, the approach scales linearly with respect to the number of atoms in terms of computational cost as well as memory, and possesses a small prefactor, making it a viable choice for large systems. In addition, it does not require any apriori knowledge of the nature or location of the instability, making it widely applicable. We verify the proposed approach by studying defect nucleation during the nanoindentation of a triangular lattice and hydrostatic tension test of an Aluminum crystal.

\section{Instability criterion}

Consider a system of $N$ atoms in a $S \in\{1,2,3\}$ dimensional space. Let $\alpha$ denote the loading parameter and $\mathbf{r}=\left[\begin{array}{llll}\mathbf{r}_{1} & \mathbf{r}_{2} & \ldots & \mathbf{r}_{N}\end{array}\right]^{T} \in \mathbb{R}^{S N \times 1}$ represent the atomic configuration. On performing a second order expansion of the system's energy $W(\alpha, \mathbf{r})$ about some configuration $\mathbf{r}^{*}$ (for fixed $\alpha$ ), we arrive at

$$
\begin{aligned}
W(\alpha, \mathbf{r}) \approx & W\left(\alpha, \mathbf{r}^{*}\right)+\mathbf{f}\left(\alpha, \mathbf{r}^{*}\right)^{T}\left(\mathbf{r}-\mathbf{r}^{*}\right) \\
& +\frac{1}{2}\left(\mathbf{r}-\mathbf{r}^{*}\right)^{T} \mathbf{H}\left(\alpha, \mathbf{r}^{*}\right)\left(\mathbf{r}-\mathbf{r}^{*}\right),
\end{aligned}
$$

where

$$
\mathbf{f}(\alpha, \mathbf{r})=\left[\begin{array}{c}
\frac{\partial W}{\partial \mathbf{r}_{1}} \\
\frac{\partial W}{\partial \mathbf{r}_{2}} \\
\vdots \\
\frac{\partial W}{\partial \mathbf{r}_{N}}
\end{array}\right] \in \mathbb{R}^{S N \times 1}
$$

represents the force on the atoms and

$$
\mathbf{H}(\alpha, \mathbf{r})=\left[\begin{array}{cccc}
\frac{\partial^{2} W}{\partial \mathbf{r}_{1}^{2}} & \frac{\partial^{2} W}{\partial \mathbf{r}_{1} \mathbf{r}_{2}} & \cdots & \frac{\partial^{2} W}{\partial \mathbf{r}_{1} \mathbf{r}_{N}} \\
\frac{\partial^{2} W}{\partial \mathbf{r}_{2} \mathbf{r}_{1}} & \frac{\partial^{2} W}{\partial \mathbf{r}_{2}^{2}} & \cdots & \frac{\partial^{2} W}{\partial \mathbf{r}_{2} \mathbf{r}_{N}} \\
\vdots & \vdots & \ddots & \vdots \\
\frac{\partial^{2} W}{\partial \mathbf{r}_{N} \mathbf{r}_{1}} & \frac{\partial^{2} W}{\partial \mathbf{r}_{N} \mathbf{r}_{2}} & \cdots & \frac{\partial^{2} W}{\partial \mathbf{r}_{N}^{2}}
\end{array}\right] \in \mathbb{R}^{S N \times S N}
$$

denotes the Hessian, also referred to as the force constants matrix.
We are interested in predicting the onset and mode of instability as the loading parameter $\alpha$ is varied. To this end, we now define the instability criterion for some given $\alpha$. Let $\mathbf{r}_{\alpha}^{*}$ represent the corresponding equilibrium atomic configuraton, i.e., $\mathbf{f}\left(\alpha, \mathbf{r}_{\alpha}^{*}\right)=\mathbf{0}$. The stability of the atomistic system can then be determined by the sign of the stability parameter

$$
\lambda_{\alpha}^{*}=\min _{\substack{\mathbf{v} \\\|\mathbf{v}\|^{2}=1}} \mathbf{v}^{T} \mathbf{H}_{\alpha}^{*} \mathbf{v},
$$

where $\|\mathbf{v}\|$ represents the $l_{2}$ norm of $\mathbf{v}$, and $\mathbf{H}_{\alpha}^{*}=$ $\mathbf{H}\left(\alpha, \mathbf{r}_{\alpha}^{*}\right)$. In particular, the system is unstable if

$$
\lambda_{\alpha}^{*}<0,
$$

which indicates that the configuration $\mathbf{r}_{\alpha}^{*}$ does not correspond to a minimum in the energy landscape, i.e., $W(\alpha, \mathbf{r})$ is not convex about $\mathbf{r}_{\alpha}^{*}$. The nature of the mode $\mathbf{v}_{\alpha}^{*}$-the minimizer of the variational problem in Eqn. 4-provides information about the type of instability, and can be used to ascertain whether it corresponds to the nucleation of a defect.

It is worth noting that in the above discussion, we do not account for additional constraints that may be prescribed on the system. For example, it is common to hold the positions of some of the atoms fixed in practical calculations. In such a situation, the components of $\mathbf{v}$ corresponding to the fixed atoms need to be enforced to be zero while solving the variational problem in Eqn. 4. Equivalently, the stability analysis can be restricted to the movable atoms by eliminating the rows and columns of $\mathbf{H}_{\alpha}^{*}$ that correspond to the fixed atoms.

\section{Lanczos framework for stability analysis}

In order to check the stability of the system at any given loading parameter $\alpha$, the variational problem in Eqn. 4 needs to be solved. The corresponding Euler-Lagrange equation is

$$
\mathbf{H}_{\alpha}^{*} \mathbf{v}=\lambda \mathbf{v},
$$

where $\lambda$ is the Lagrange multiplier used to enforce the constraint. It follows that the lowest eigenvalue and corresponding eigenvector of $\mathbf{H}_{\alpha}^{*}$ coincide with the stability parameter $\lambda_{\alpha}^{*}$ and mode $\mathbf{v}_{\alpha}^{*}$, respectively. Instead of solving for $\lambda_{\alpha}^{*}$ 
and $\mathbf{v}_{\alpha}^{*}$ alone, it is common to eigendecompose $\mathbf{H}_{\alpha}^{*}[19,20,21,22]$, i.e., compute all its eigenvalues and eigenvectors. However, the computational and memory costs of such a procedure scale as $\mathcal{O}\left(N^{3}\right)$ and $\mathcal{O}\left(N^{2}\right)$ with respect to the number of atoms, respectively, which severely restricts the size of systems that can be studied. In order to overcome this limitation, it has been proposed that the stability analysis be restricted to a suitably chosen subset of atoms [23, 24, 25]. However, such techniques assume $\mathbf{v}_{\alpha}^{*}$ to be localized, and even then they need apriori knowledge of the location and size of the localization region to be efficient. In addition, they require the original ordering of the eigenvalues to remain unaffected by the localization. Such assumptions/approximations can lead to inaccurate results, as demonstrated by the results in Section 4.

In view of the above discussion, we propose calculating $\lambda_{\alpha}^{*}$ and $\mathbf{v}_{\alpha}^{*}$ using the implicitly restarted [31] version of the Lanczos method [32] outlined in Algorithm 1. In the Lanczos method, a three term recurrence relation is utilized to generate an orthogonal basis $\mathbf{v}_{k}$ in which $\mathbf{H}_{\alpha}^{*}$ is a tridiagonal matrix:

$$
\mathbf{J}_{k}=\left[\begin{array}{ccccc}
a_{1} & b_{1} & & & \\
b_{1} & a_{2} & b_{2} & & \\
& \ddots & \ddots & \ddots & \\
& & b_{k-2} & a_{k-1} & b_{k-1} \\
& & & b_{k-1} & a_{k}
\end{array}\right] \in \mathbb{R}^{k \times k},
$$

where $a_{k}$ and $b_{k}$ are scalars calculated during the iteration. The eigenvalues of $\mathbf{J}_{k}$ approximate those of $\mathbf{H}_{\alpha}^{*}$, with the algebraically smallest and largest ones being the first to converge. Thereafter, the transformation matrix $\mathbf{V}$ consisting of the Lanczos vectors $\mathbf{v}_{k}$ as columns is used to determine the eigenmode $\mathbf{v}_{\alpha}^{*}$. In this work, rather than explicitly form the matrix $\mathbf{H}_{\alpha}^{*}$, we utilize the finite-difference approximation for the product of $\mathbf{H}_{\alpha}^{*}$ with any vector $\mathbf{w}$ :

$$
\mathbf{H}^{*} \mathbf{w} \approx \frac{\mathbf{f}\left(\alpha, \mathbf{r}_{\alpha}^{*}+\varepsilon \mathbf{w}\right)-\mathbf{f}\left(\alpha, \mathbf{r}_{\alpha}^{*}\right)}{\varepsilon},
$$

where $\varepsilon$ is an appropriately small parameter. In doing so, each matrix-vector product is replaced with one force evaluation, since $\mathbf{f}\left(\alpha, \mathbf{r}_{\alpha}^{*}\right)$ is readily available. Such a strategy is attractive because of the substantial reduction in the computational cost and memory storage requirements, particularly when the interactions are relatively long-ranged. In addition, since the second order derivatives of the energies are not required, the exact same implementation can be interfaced with any flavor of atomistic simulation, including those where the second derivatives are hard/expensive to evaluate, e.g. electronic structure calculations.

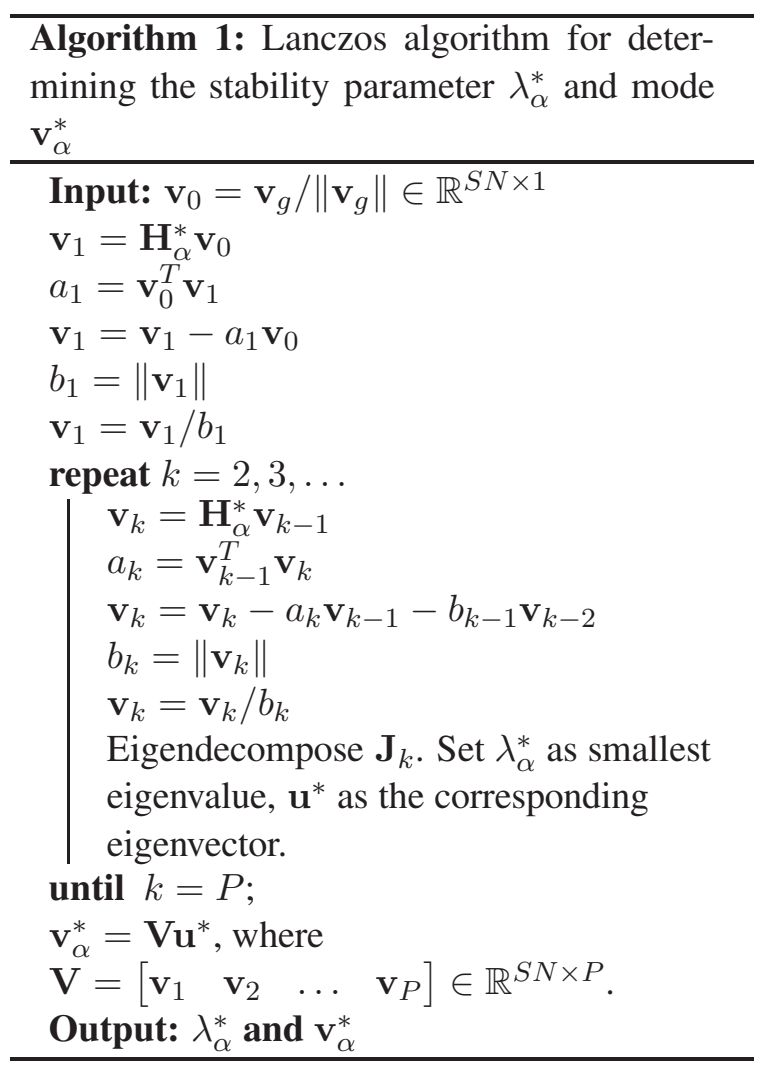

The potentials employed in atomistic simulations are typically short-ranged, whereby $\mathbf{H}_{\alpha}^{*}$ is a sparse matrix whose sparsity increases with system size. Furthermore, the number of iterations required for convergence to a prespecified tolerance is independent of the size of $\mathbf{H}_{\alpha}^{*}$ [33], which makes the overall computational and memory costs scale as $\mathcal{O}(N)$ with respect to the number of atoms. In fact, this scaling becomes independent of system size when $\mathbf{v}_{\alpha}^{*}$ is localized and a good starting guess $\mathbf{v}_{g}$ is provided. Notably, if Newton's method is used in conjunction with the minimum residual (MINRES) method [34], $\lambda_{\alpha}^{*}$ and $\mathbf{v}_{\alpha}^{*}$ can be calculated with practically zero computational effort in addition to that required for determining the equilibrium position 
of the atoms. This is possible because the Lanczos vectors $\mathbf{v}_{k}$ are generated as part of the MINRES algorithm, whereby $\lambda_{\alpha}^{*}$ and $\mathbf{v}_{\alpha}^{*}$ can be estimated using the data from the last linear solve. Indeed, the $\lambda_{\alpha}^{*}$ and $\mathbf{v}_{\alpha}^{*}$ so determined can be further refined by setting $\mathbf{v}_{g}=\mathbf{v}_{\alpha}^{*}$ as the starting guess for the Lanczos method, which should then converge extremely rapidly.

The Lanczos framework as presented above is suitable for calculating the lowest eigenvalue and corresponding eigenvector of $\mathbf{H}_{\alpha}^{*}$. However, several lowest eigenvalues and corresponding eigenvectors may be required in certain situations, e.g. post-bifurcation stability analysis $[21,22]$. The Lanczos method in Algorithm 1 is applicable to such situations, albeit with minor modifications. It is also possible that higher order derivatives of the energy may be needed while performing asymptotic analysis near critical points, e.g. Lyapunov-Koiter-Schmidt decomposition [35]. In such situations, it is envisioned that the finite-difference approximation in Eqn. 8 can be suitably generalized.

\section{Examples and results}

In this section, we verify the accuracy and efficiency of the proposed approach for predicting defect nucleation in atomistic systems. In all the simulations, we use the Lanczos method [32] described in Algorithm 1 in conjunction with implicit restarts [31]. We choose $\varepsilon=10^{-6}$ for approximating the product of $\mathbf{H}_{\alpha}^{*}$ with any vector. For modeling the interaction between atoms in a triangular lattice, we utilize the Lennard-Jones potential [36]:

$\mathcal{E}_{L J}(r)=\left\{\begin{array}{ll}\epsilon_{0}\left[\left(\frac{\sigma}{r}\right)^{12}-2\left(\frac{\sigma}{r}\right)^{6}\right] & \text { if } r \leq r_{c} \\ 0 & \text { if } r>r_{c}\end{array}\right.$,

with the parameters $\epsilon_{0}=1 \mathrm{eV}, \sigma=1.1225 \AA$ and $r_{c}=2.5 \AA$, which results in a equilibrium lattice constant of $a_{0}=1.1327 \AA$. For modeling the interaction of Aluminum atoms in a face-centered cubic (FCC) lattice, we employ the Morse approximation to the Lennard-Jones potential [37]:

$\mathcal{E}_{M}(r)=\left\{\begin{array}{ll}\epsilon_{0}\left[e^{-12 \frac{(r-\sigma)}{\sigma}}-2 e^{-6 \frac{(r-\sigma)}{\sigma}}\right] & \text { if } r \leq r_{c} \\ 0 & \text { if } r>r_{c}\end{array}\right.$,

(10) with the parameters $\epsilon_{0}=0.17 \mathrm{eV}, \sigma=2.92 \AA$, and $r_{c}=10 \AA$, which results in a equilibrium lattice constant of $a_{0}=4.0572 \AA$. During geometry optimization, we determine the equilibrium position of atoms using the trust-region dogleg algorithm [38] with tolerances of $1 \times 10^{-12} \mathrm{eV}$ and $1 \times 10^{-12} \mathrm{eV} / \AA$ for the energy and forces, respectively. The tolerance for the energy represents the normalized change in the total energy, whereas the tolerance for the force denotes the maximum force on any atom.

\subsection{Accuracy and convergence}

We first verify the convergence of the proposed approach to the machine precision accurate result. For this study, we consider a FCC Aluminum lattice at the bulk lattice constant of $a_{0}=4.0572 \AA$ with the atomic interactions governed by the Morse approximation potential in Eqn. 10. We subject the lattice to a hydrostatic stretch of $\eta=1.13$, at which point the system is unstable (Section 4.2). In Fig. 1, we plot the convergence of $\lambda_{\alpha}^{*}$ and $\mathbf{v}_{\alpha}^{*}$ during the Lanczos iteration - starting from a random guess $\mathbf{v}_{g}$-for systems consisting of 5324 and 10976 atoms. Since $\lambda_{\alpha}^{*}$ is three-fold degenerate, we utilize the subspace spanned by the three corresponding eigenvectors to check for the convergence of $\mathbf{v}_{\alpha}^{*}$. Specifically, we define the error in $\mathbf{v}_{\alpha}^{*}$ to be the angle between the computed and converged subspaces. We observe that there is rapid convergence in both $\lambda_{\alpha}^{*}$ and $\mathbf{v}_{\alpha}^{*}$, with an accuracy of even $10^{-6}$ attained in modest number of force evaluations. Overall, we conclude that the proposed method can accurately determine the stability parameter $\lambda_{\alpha}^{*}$ and the corresponding mode $\mathbf{v}_{\alpha}^{*}$.

\subsection{Examples \\ 4.2.1. Nanoindentation of a triangular lattice}

We now study the nucleation of a dislocation during nanoindentation [39] using the LAMMPS [40] framework. Specifically, we consider a triangular lattice with bulk lattice constant of $1.1327 \AA$ in a rectangular domain of size $45.308 \times 19.619 \AA$, the origin being located at the bottom left corner. We hold the bottom two rows of atoms fixed throughout the simulation, and enforce periodic boundary conditions on the lateral sides of the domain, i.e., on the edges cor- 


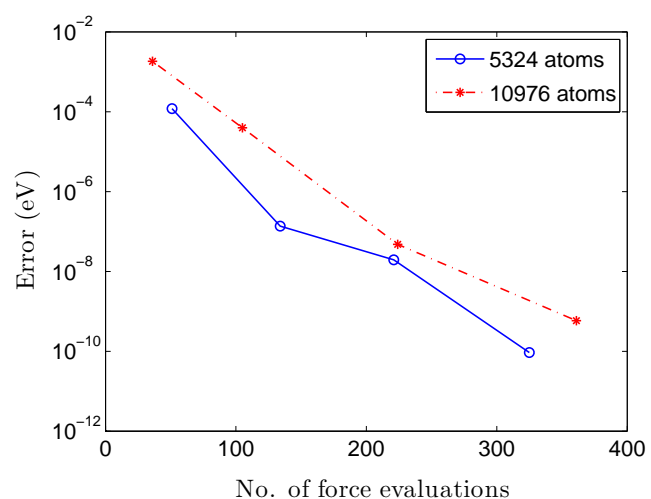

(a) Stability parameter $\lambda_{\alpha}^{*}$

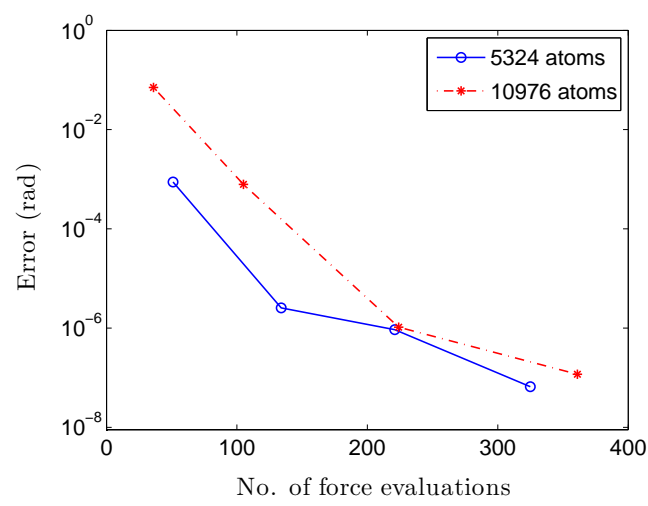

(b) Mode $\mathbf{v}_{\alpha}^{*}$

Figure 1: Convergence of the stability parameter $\lambda_{\alpha}^{*}$ and mode $\mathbf{v}_{\alpha}^{*}$ during the implicitly restarted Lanczos iteration. The error in the three-fold degenerate $\lambda_{\alpha}^{*}$ denotes the absolute value of the difference from the converged value. The error in $\mathbf{v}_{\alpha}^{*}$ corresponds to the angle between the subspaces spanned by the computed and converged eigenvectors corresponding to $\lambda_{\alpha}^{*}$.

We model the spherical indenter with a repulsive force of the form [41]

$$
\mathcal{F}(r)=\left\{\begin{array}{ll}
-K(R-r)^{2} & \text { if } r \leq R \\
0 & \text { if } r>R
\end{array},\right.
$$

where $r$ is the distance from the center of the indenter, $R$ is the radius of the indenter and $K$ is the strength of the indenter. In this study, we choose the parameters $R=9.8095 \AA$ and $K=882.84$ $\mathrm{eV} / \AA^{3}$.

We start with the center of the indenter at $(22.6540,25.5047) \AA$, and displace it in the negative $y$ direction in steps of $1.9619 \times 10^{-3} \AA$ until $\lambda_{\alpha}^{*}$ becomes negative. Then, starting from the previous step, the step size is reduced by half and the procedure is repeated until the indentation depth at which the instability is predicted is accurate to within $5 \times 10^{-4} \AA$. Subsequently, the indentation is continued from the indentation depth at which the first negative $\lambda_{\alpha}^{*}$ was encountered with the original step size of $1.9619 \times 10^{-3} \AA$. At this point, the mode $\mathbf{v}_{\alpha}^{*}$ corresponding to the the onset of instability is used to generate a good starting guess so as to converge to a minimum energy configuration. In Fig. 2, we plot the stability parameter $\lambda_{\alpha}^{*}$ as a function of the indenter displacement. We observe that there is a steady increase in $\lambda_{\alpha}^{*}$ during the initial stages of nanoindentation, before it undergoes an extremely sharp drop close to the point of instability, which occurs at an indenter displacement of $1.5474 \AA$. As shown in Fig. 3, the minimum energy configurations after the point of instability show the presence of a dislocation. Therefore, we expect the instability mode $\mathbf{v}_{\alpha}^{*}$ to predict the nucleation of a dislocation, a result we verify next.

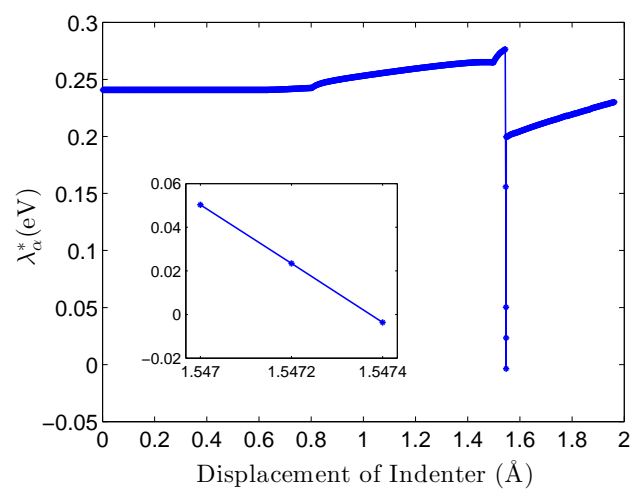

Figure 2: Variation of the stability parameter $\lambda_{\alpha}^{*}$ during the nanoindentation test. The inset magnifies the plot close to the point of instability.

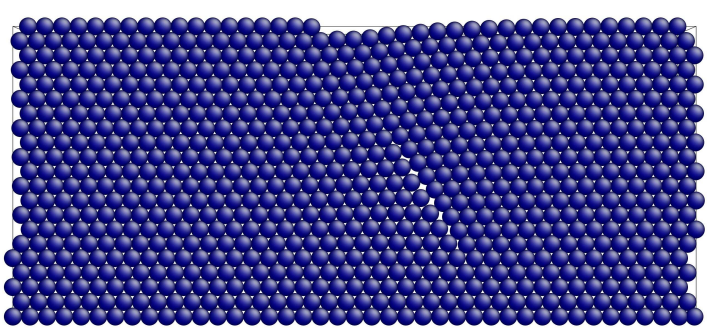

Figure 3: Minimum energy configuration subsequent to the onset of instability during the nanoindentation test. Figure generated using AtomEye [42]

Since $\mathbf{v}_{\alpha}^{*}$ and $-\mathbf{v}_{\alpha}^{*}$ are two equivalent mini- 
mizers of the variational problem in Eqn. 4, we present in Fig. 4 the configurations obtained by adding and subtracting $\mathbf{v}_{\alpha}^{*}$ to the unstable configuration. We observe dislocations nucleating from the surface, with slip predicted on either of the two possible slip planes. This is to be expected since both slip configurations are equally probable based on symmetry considerations. In practical computations, one configuration is picked over the other due to the presence of round-off errors. Indeed, the configuration obtained by subtracting $\mathbf{v}_{\alpha}^{*}$ is in very good agreement with the numerical result presented in Fig. 3. The need for $\mathbf{v}_{\alpha}^{*}$ to be able to predict both symmetry related slip configurations indicates that the trajectory followed by atoms through configurational space is not just one of pure slip. In order to verify this, we plot the contours of the instability mode $\mathbf{v}_{\alpha}^{*}$ in Fig. 5. We also plot $\mathbf{v}_{\alpha}^{*}$ in the vicinity of the indenter in Fig. 6. It is clear from these plots that $\mathbf{v}_{\alpha}^{*}$ is not very localized and in fact has significant components on both the slip planes. Therefore, a localized stability analysis that is restricted to only one of the slip planes is likely to produce inaccurate results.

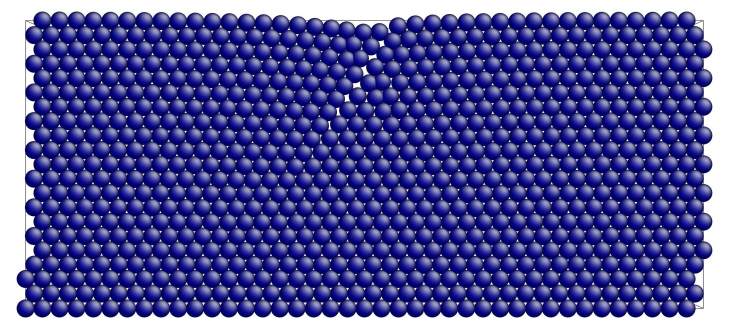

(a) Unstable configuration plus $\mathbf{v}_{\alpha}^{*}$

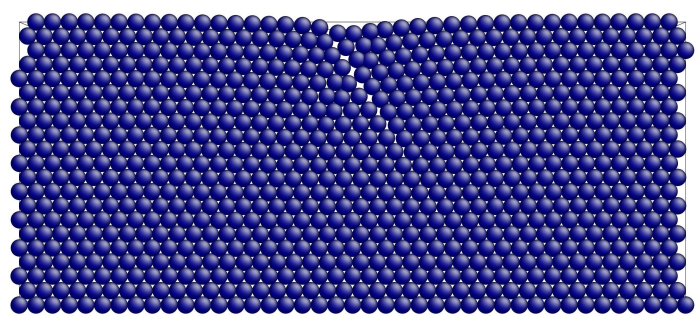

(b) Unstable configuration minus $\mathbf{v}_{\alpha}^{*}$

Figure 4: Configurations generated by adding and subtracting $\mathbf{v}_{\alpha}^{*}$ at the onset of instability during the nanoindentation test. Figures generated using AtomEye [42]

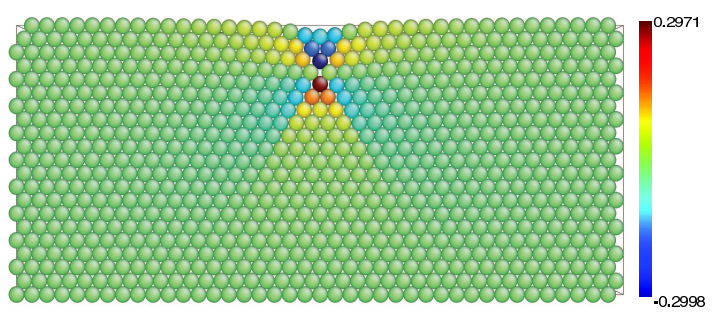

(a) $x$ component of $\mathbf{v}_{\alpha}^{*}$

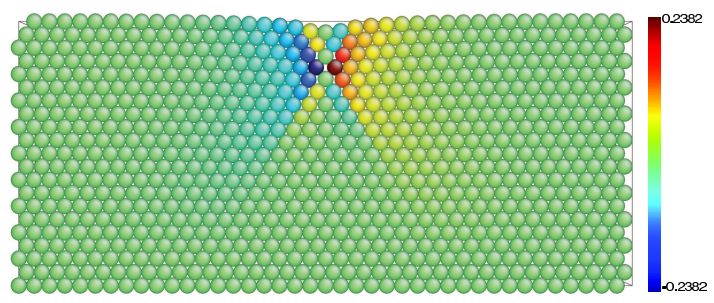

(b) $y$ component of $\mathbf{v}_{\alpha}^{*}$

Figure 5: Contours of $\mathbf{v}_{\alpha}^{*}$ at the onset of instability during the nanoindentation test. Figures generated using AtomEye [42]

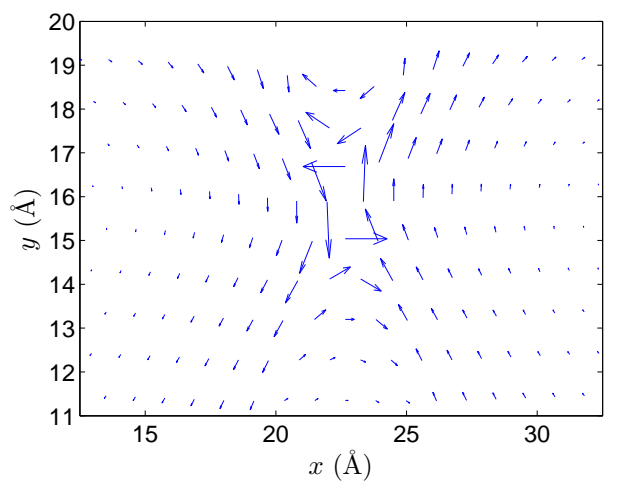

Figure 6: Instability mode $\mathbf{v}_{\alpha}^{*}$ in the vicinity of the indenter during the nanoindentation test.

\subsubsection{Hydrostatic tension test of Aluminum}

We next study the nucleation of defects during the hydrostatic tension test of a FCC Aluminum crystal. Specifically, we consider a system of 27,436 atoms interacting via the Morse approximation potential in Eqn. 10. The atoms on the boundary are held fixed to prevent any rigid body modes. Starting from the original unstressed state, we apply hydrostatic stretches $\eta$ in steps of 0.01 until $\lambda_{\alpha}^{*}$ becomes negative. Subsequently, starting from the previous step, the step size is reduced by half and the procedure is re- 
dicted to within $2 \times 10^{-4}$. In Fig. 7, we plot the variation of the stability parameter during the aforedescribed hydrostatic tension test. We observe that $\lambda_{\alpha}^{*}$ reduces gradually, with the onset of instability predicted at $\eta=1.1214$. At this point, the stability parameter $\lambda_{\alpha}^{*}$ is three-fold degenerate, and therefore any linear combination of the corresponding eigenvectors is a valid mode $\mathbf{v}_{\alpha}^{*}$. The instability modes plotted in Fig. 8 appear to predict the formation of multiple voids in the homogeneous lattice.

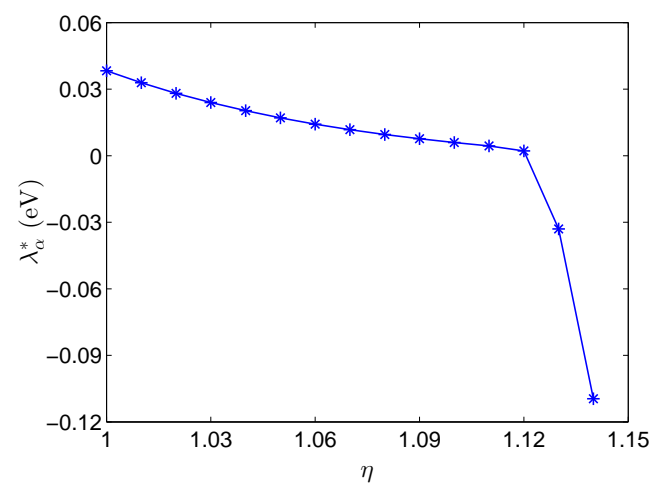

Figure 7: Stability parameter $\lambda_{\alpha}^{*}$ as a function of the stretch $\eta$ during the hydrostatic tension test.

We now investigate the effect of localization on the results presented above. On restricting the stability analysis to atoms within a sphere of radius $10 \AA$ centered at the midpoint of the domain, we find that $\lambda_{\alpha}^{*}$ is no longer degenerate at the point of instability. In addition, the corresponding mode $\mathbf{v}_{\alpha}^{*}$ plotted in Fig. 9 appears to predict the formation of a single large void, which is in agreement with the conclusions drawn from a prior localized stability analysis [24]. However, it is clear that these results are very different to those obtained for the full system. Indeed, the results from the localized analysis approach those obtained for the full system as the localization region is increased. This highlights the need for a complete stability analysis in order to accurately predict defect nucleation in atomistic systems.

\subsection{Scaling and performance}

Finally, we study the scaling and performance of the proposed Lanczos based method and compare it with the QR approach, an efficient technique for eigendecomposition. To ensure a fair comparison, rather than employing a finitedifference approximation for the matrix-vector

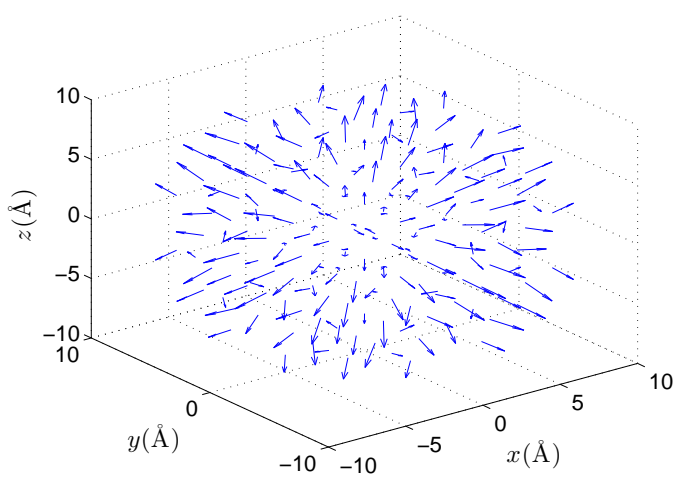

(a) First degenerate mode

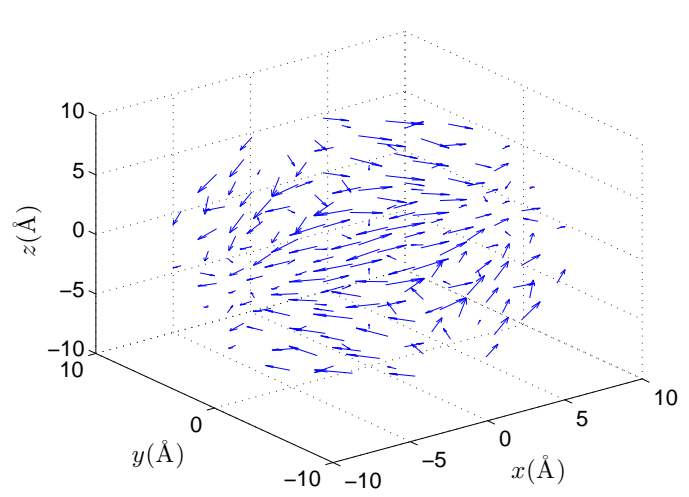

(b) Second degenerate mode

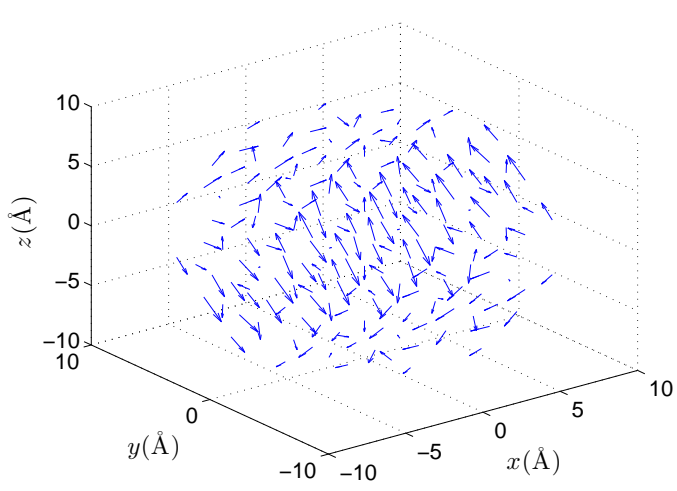

(c) Third degenerate mode

Figure 8: The instability modes corresponding to the threefold degenerate stability parameter $\lambda_{\alpha}^{*}$ during the hydrostatic tension test. Only the components in a spherical subdomain of radius $10 \AA$ centered at the origin have been plotted.

product in the Lanczos framework, we use the full matrix $\mathbf{H}_{\alpha}^{*}$. We again choose a FCC Aluminum lattice subjected to a hydrostatic stretch of $\eta=1.13$ as the representative example. In Fig. 


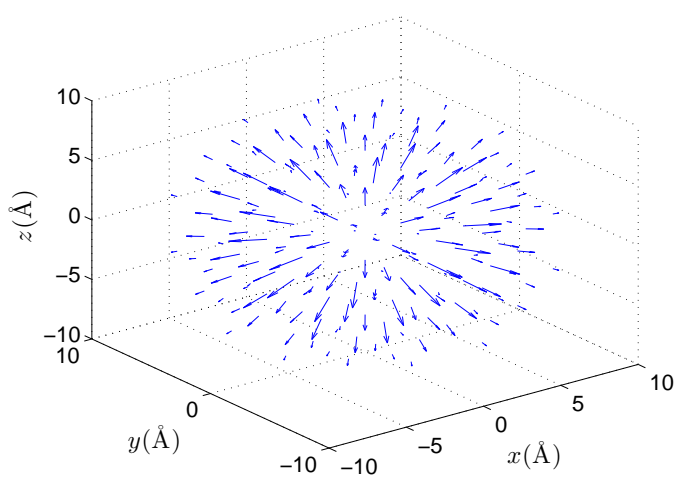

Figure 9: Instability mode $\mathbf{v}_{\alpha}^{*}$ obtained during the hydrostatic tension test by restricting the stability analysis to a spherical subdomain of radius $10 \AA$ centered at the midpoint of the domain.

an accuracy of $10^{-5} \mathrm{eV}$ in $\lambda_{\alpha}^{*}$ and $10^{-5} \mathrm{rad}$ in $\mathbf{v}_{\alpha}^{*}$ for systems ranging from around 1000 to $1,000,000$ atoms, starting from a random starting guess $\mathbf{v}_{g}$. For reference, we also plot lines corresponding to $\mathcal{O}(N)$ and $\mathcal{O}\left(N^{3}\right)$ scaling with respect to the number of atoms. Notably, the QR results are restricted to 5324 atoms due to the tremendous computational cost and memory requirements for larger systems. We observe that the proposed Lanczos based approach is able to significantly outperform the QR method. Moreover, it demonstrates $\mathcal{O}(N)$ scaling with system size compared to the $\mathcal{O}\left(N^{3}\right)$ scaling of the QR method. In fact, the time taken by the Lanczos method for the 1,000,000 atom system is approximately 7 minutes on a single workstation. Indeed, the computational times are expected to be much smaller in practical simulations due to the availability of a good guess. Combined with its extremely good parallel scalability, the proposed approach is suitable for the stability analysis of atomistic systems even at the mesoscale.

\section{Concluding remarks}

We have presented an accurate and efficient approach based on the Lanczos algorithm for predicting the onset and mode of instability in atomistic systems. Specifically, we have developed a computational framework-identically applicable to all flavors of atomistic simulations, including electronic structure calculations-wherein we have not made any apriori assumptions regarding the nature of the instability or its loca-

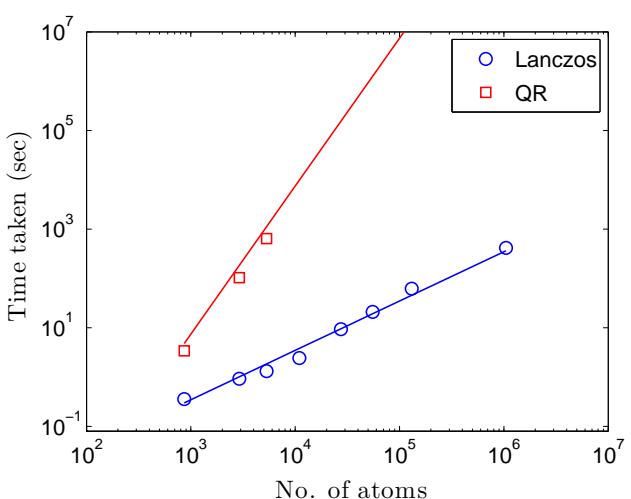

Figure 10: Comparison of the proposed Lanczos based method with eigendecomposition using the QR method. The solid lines represent $\mathcal{O}(N)$ and $\mathcal{O}\left(N^{3}\right)$ scaling with respect to number of atoms.

tion. We have verified the accuracy of the proposed method by studying two examples involving defect nucleation, namely the nanoindentation of a triangular lattice and the hydrostatic tension test of an Aluminum crystal. We have demonstrated that the computational cost scales linearly with the number of atoms-accompanied by a small prefactor-in practical computations. Overall, we conclude that the proposed approach makes it feasible to routinely study the stability of atomistic systems at the mesoscale.

\section{Acknowledgements}

The authors gratefully acknowledge the support of National Science Foundation under Grant Number 1333500. The authors are also thankful for the valuable comments and suggestions of the anonymous referees.

\section{References}

[1] R. Phillips, Crystals, defects and microstructure: modeling across scales, Cambridge University Press, 2001.

[2] P. Suryanarayana, K. Bhattacharya, M. Ortiz, Journal of the Mechanics and Physics of Solids 61 (2013) 3860.

[3] P. Suryanarayana, V. Gavini, T. Blesgen, K. Bhattacharya, M. Ortiz, Journal of the Mechanics and Physics of Solids 58 (2010) 256-280.

[4] P. Suryanarayana, K. Bhattacharya, M. Ortiz, Journal of Computational Physics 230 (2011) 5226-5238.

[5] P. Motamarri, M. R. Nowak, K. Leiter, J. Knap, V. Gavini, Journal of Computational Physics 253 (2013) 308-343.

[6] A. S. Banerjee, R. S. Elliott, R. D. James, Journal of Computational Physics 287 (2015) 226-253. 
[7] S. Ghosh, P. Suryanarayana, Journal of Computational Physics 307 (2016) 634-652.

[8] S. Ghosh, P. Suryanarayana, arXiv preprint arXiv:1603.04334 (2016).

[9] O. R. de la Fuente, J. Zimmerman, M. Gonzalez, J. De la Figuera, J. Hamilton, W. W. Pai, J. Rojo, Physical review letters 88 (2002) 036101.

[10] E. Lilleodden, J. Zimmerman, S. Foiles, W. Nix, Journal of the Mechanics and Physics of Solids 51 (2003) 901-920.

[11] R. E. Miller, L. Shilkrot, W. A. Curtin, Acta Materialia 52 (2004) 271-284.

[12] F. Cleri, D. Wolf, S. Yip, S. Phillpot, Acta materialia 45 (1997) 4993-5003.

[13] T. Zhu, J. Li, S. Yip, Physical review letters 93 (2004) 025503.

[14] M. Tschopp, G. Tucker, D. McDowell, Computational Materials Science 44 (2008) 351-362.

[15] R. E. Rudd, J. F. Belak, Computational materials science 24 (2002) 148-153.

[16] J. Wang, R. Hoagland, J. Hirth, L. Capolungo, I. Beyerlein, C. Tomé, Scripta Materialia 61 (2009) 903906.

[17] M. S. Wu, K. Zhou, A. A. Nazarov, Phys. Rev. B 76 (2007) 134105

[18] A. Garg, A. Acharya, C. E. Maloney, Journal of the Mechanics and Physics of Solids 75 (2015) 76-92.

[19] T. Kitamura, Y. Umeno, R. Fushino, Materials Science and Engineering: A 379 (2004) 229-233.

[20] T. Kitamura, Y. Umeno, N. Tsuji, Computational materials science 29 (2004) 499-510.

[21] R. S. Elliott, N. Triantafyllidis, J. A. Shaw, Journal of the Mechanics and Physics of Solids 54 (2006) 161192

[22] R. S. Elliott, J. A. Shaw, N. Triantafyllidis, Journal of the Mechanics and Physics of Solids 54 (2006) 193232

[23] R. E. Miller, D. Rodney, Journal of the Mechanics and Physics of Solids 56 (2008) 1203-1223.

[24] T. Delph, J. Zimmerman, J. Rickman, J. Kunz, Journal of the Mechanics and Physics of Solids 57 (2009) 6775.

[25] T. Delph, J. Zimmerman, Modelling and Simulation in Materials Science and Engineering 18 (2010) 045008.

[26] K. J. Van Vliet, J. Li, T. Zhu, S. Yip, S. Suresh, Physical Review B 67 (2003) 104105.

[27] J. Li, K. J. Van Vliet, T. Zhu, S. Yip, S. Suresh, Nature 418 (2002) 307-310.

[28] J. Li, T. Zhu, S. Yip, K. J. Van Vliet, S. Suresh, Materials Science and Engineering: A 365 (2004) 25-30.

[29] T. Delph, Proceedings of the Royal Society A: Mathematical, Physical and Engineering Science 461 (2005) 1869-1888.

[30] N. C. Admal, E. B. Tadmor, Journal of Elasticity 100 (2010) 63-143.

[31] R. B. Lehoucq, D. C. Sorensen, SIAM Journal on Matrix Analysis and Applications 17 (1996) 789-821.

[32] C. Lanczos, An iteration method for the solution of the eigenvalue problem of linear differential and integral operators, United States Governm. Press Office, 1950.

[33] Y. Saad, Numerical methods for large eigenvalue problems, volume 158, SIAM, 1992.
[34] C. C. Paige, M. A. Saunders, SIAM journal on numerical analysis 12 (1975) 617-629.

[35] R. Peek, M. Kheyrkhahan, Computer methods in applied mechanics and engineering 108 (1993) 261-279.

[36] D. A. McQuarrie, J. D. Simon, Physical chemistry: a molecular approach, University Science Books, 1997.

[37] T.-C. Lim, Zeitschrift für Naturforschung A 58 (2003) 615-617.

[38] M. J. Powell, A FORTRAN subroutine for solving systems of nonlinear algebraic equations., Technical Report, Atomic Energy Research Establishment, Harwell (England), 1968.

[39] A. C. Fischer-Cripps, Nanoindentation, volume 1, Springer, 2011.

[40] S. Plimpton, Journal of Computational Physics 117 (1995) $1-19$.

[41] E. Njeim, D. Bahr, Scripta Materialia 62 (2010) 598601.

[42] J. Li, Modelling and Simulation in Materials Science and Engineering 11 (2003) 173. 